www.jmscr.igmpublication.org Impact Factor 5.244

Index Copernicus Value: 83.27 ISSN (e)-2347-176x ISSN (p) 2455-0450 crossref DOI: _https://dx.doi.org/10.18535/jmscr/v4i12.67

\title{
Comparative Study of Liver Function Parameters in Patients with Diabetes and Hypertension
}

\author{
Authors \\ Dr Kumar Prafull Chandra, Dr. Dhirendra Kumar Shukla, Dr. Anil Kumar Pawah \\ GCRG Institute \\ Corresponding Author \\ Dr K P Chandra \\ Consultant Physician
}

1/15; Vinamra khand, Gomtinagar near Kathauta Chauraha, Lucknow UP, Pin 226010

Email: drkpchandra@gmail.com

\begin{abstract}
Background: Abnormality in liver function test parameters in patients with type 2 diabetes mellitus (T2DM) is common, but study comparing this association in patients of diabetes with or without hypertension is lacking.

Aims and Objective: To compare the liver function test parameters and its correlation with the lipids parameters in patients with T2DM and hypertension with diabetes.

Materials and Methods: A prospective study was done including 141 diabetic patients in the Department of Medicine, Mayo Institute of Medical sciences, Barabanki between February 2016 to June 2016. Patients were divided in to Group D ( $n=42$, patients having diabetes) and Group HD ( $n=99$, hypertensive diabetes mellitus). Patients were also divided on the basis of extend of glycemic control as good control (HbAlc $<7 \%)$, poor control (HbAlc between 7-9\%) and uncontrolled (HbAlc >9). Detailed demographic, glycemic, lipid and thyroid function test parameters were estimated in all the study population. Data collected was analysed using SPSS ver. 20 and $p$ value of $<0.05$ was considered as significant.

Results: Out of 141 patients, 42 (29.78\%) were having diabetes alone, whereas 99 (70.21\%) were having hypertension along with diabetes. All the demographic parameters except age $(p=0.0003)$ were comparable. Glycemic parameters such as fasting plasma glucose $(p>0.05)$ and post prandial blood glucose $(p>0.05)$ were comparable except HbAlc $(p=0.021)$ and average blood glucose $(p=0.022)$ between both the groups. All the liver function test parameters including alkaline phosphatase, bilirubin direct, bilirubin total, bilirubin indirect, gamma-glutamyl transpeptidase (GGT), aspartate aminotransferase (SGOT) and alanine aminotransferase (SGPT) were insignificantly higher in patients with diabetes as compared to patients having diabetes with hypertension ( $p>0.05$ ). However, bilirubin direct, GGT, SGOT and SGPT were higher than the normal range in patients with diabetes. Alkaline Phosphate, GGT, SGOT and SGPT were also significantly higher in patients with uncontrolled diabetes as compared to patients with good control $(p<0.05)$.

Conclusion: Liver function test parameters such as bilirubin direct, GGT, SGOT and SGPT were higher in patients with T2DM, but comparable with patients with hypertensive diabetics. $(p>0.05)$. Alkaline Phosphate, GGT, SGOT and SGPT were higher in patients with uncontrolled diabetes.

Keywords: liver function test, T2DM, hypertension.
\end{abstract}




\section{Introduction}

Screening of liver function tests (LFTs) for liver disease in clinical practice is commonly used by the physician. Liver function tests are also being used to monitor progression of known disease and also to monitor the adverse effects caused by hepatotoxic drugs. 1,2

Most commonly used LFTs in clinical practice are alkaline phosphatase, serum aminotransferases and bilirubin. Two very important aminotransferases such as alanine aminotransferase (ALT) and aspartate aminotransferase (AST) are the measure of the intracellular hepatic enzymes that have leaked out in to systemic circulation. These markers serve as a marker of hepatocytes injury. ${ }^{2}$

Association between diabetes mellitus and liver injury is well established. Carbohydrate homeostasis is also regulated by liver. In patients with hyperglycemia, intracellular glycogen is increased in hepatocytes in response to increase glycogen synthesis. ${ }^{3}$ In response to this, there is a mild to moderate increase in aminotransferases. This abnormality was found to be reversed by the good glycemic control. ${ }^{4}$

Hypertension with diabetes is a deadly duo; high blood pressure (hypertension) can result in to worsening many diabetes related complications such as eye and kidney disease. ${ }^{5,6}$ Reports have also shown that most of people with diabetes develop hypertension during their life. ${ }^{7}$

We have not found any study comparing the liver function tests in patients with diabetes and hypertension diabetes. Hence, in present study we tried to find out the difference of liver function tests between patients with diabetes and hypertension diabetes.

\section{Materials and Methods}

A prospective study was performed on 141 diabetic patients in the Department of Medicine, Mayo Institute of Medical sciences, Barabanki for 6 months from February 2016.

All the patients were divided in to Group D ( $n=42$, patients having diabetes) and Group HD ( $n=99$, hypertensive diabetics). Patients were further divided on the basis of extend of glycemic control as good control (HbA1c <7\%), Poor Control (HbA1c between 7-9\%) and Uncontrolled (HbA1c $>9 \%$ ) to compare liver function test parameters between these groups.

A written Informed consent from all patients and Institutional Ethics Committee approval was obtained before starting the study.

Patients with confirmed T2DM or newly diagnosed T2DM as per the criteria set by American Diabetes Association (ADA) 2016, fasting plasma glucose $(\mathrm{FPG}) \geq 126 \mathrm{mg} / \mathrm{dl}$, or random or two hour post prandial plasma glucose (PPG) of $\geq 200 \mathrm{mg} / \mathrm{dl}$ were included in the present study.

Patients with diabetes having history of alcohol intake, hepatotoxic drugs such as amiodarone and antituberculous drugs, having history of any liver disease, acute hepatitis and having any form of evidence of hepatitis B and C virus infection were excluded from the present study.

Demographic parameters such as age, height, weight and body mass index (BMI) were recorded for all the patients. Glycemic parameters such as fasting plasma glucose (FPG), post prandial glucose (PPG), HbA1c and average blood glucose was estimated after drawing blood from all the patients. Estimation of parameters deciding liver function such as Alkaline Phosphate, Bilirubin Direct, Bilirubin Total, Bilirubin Indirect, Gamma-Glutamyl Transpeptidase (GGT), aspartate aminotransferase (SGOT) and alanine aminotransferase (SGPT) were estimated for each patient under study.

All the statistical analysis was performed using IBM SPSS ver. 20. Mean and standard deviations were calculated for time varying variables and percentages were calculated for categorical variables. $\mathrm{P}$ value $<0.05$ was considered as significant.

\section{Results}

Out of 141 patients, $42(29.78 \%)$ were having diabetes only whereas $99 \quad(70.21 \%)$ were hypertensive diabetes. There were $34(80.95 \%)$ male and $8(19.05 \%)$ female in patients with 
diabetes whereas there were $76(76.76 \%)$ male and $23(23.23 \%)$ female who were hypertensive diabetes.

Table 1: Demographic parameters between diabetes and hypertensive diabetes patients

\begin{tabular}{|l|c|c|c|}
\hline Variable & DM $(\mathbf{n = 4 2})$ & DM+HTN $(\mathbf{n = 9 9 )}$ & P value \\
\hline Age $($ year $)$ & $42.24 \pm 12.02$ & $53.77 \pm 11.15$ & 0.0003 \\
\hline Height $(m e t e r)$ & $6.26 \pm 23.46$ & $4.85 \pm 21.34$ & NS \\
\hline Weight $(\mathrm{kg})$ & $68.52 \pm 11.64$ & $71.34 \pm 11.46$ & NS \\
\hline BMI $\left(\mathrm{kg} / \mathrm{m}^{2}\right)$ & $22.63 \pm 5.30$ & $25.66 \pm 5.72$ & NS \\
\hline FBS $(\mathrm{mg} / \mathrm{dl})$ & $132.68 \pm 42.94$ & $138.44 \pm 41.29$ & NS \\
\hline PPG $(\mathrm{mg} / \mathrm{dl})$ & $242.16 \pm 91.67$ & $232.85 \pm 76.10$ & NS \\
\hline HbA1c $(\%)$ & $9.21 \pm 2.24$ & $8.11 \pm 1.83$ & 0.021 \\
\hline ABG $(\mathrm{mg} / \mathrm{dl})$ & $218.41 \pm 70.55$ & $190.08 \pm 51.33$ & 0.022 \\
\hline
\end{tabular}

Data is expressed as mean \pm SD, DM+ HTN; diabetic hypertensive, DM; diabetes, BMI; body mass index, FBG; fasting blood glucose, PPG; post prandial glucose, HbA1c; glycated hemoglobin, ABG; average blood glucose calculated from HbA1c values, $\mathrm{P}$ value $<0.05$ is considered as significant.

Table 2: Comparison of liver function parameters between diabetes and hypertensive diabetes

\begin{tabular}{|c|c|c|c|c|}
\hline Variable & $\begin{array}{c}\text { Group D } \\
(n=42)\end{array}$ & $\begin{array}{c}\text { Group HD } \\
(n=99)\end{array}$ & $\begin{array}{c}\text { Reference } \\
\text { range }^{8}\end{array}$ & $P$ value \\
\hline Alkaline Phosphatase & $123.35 \pm 63.98$ & $104.83 \pm 41.36$ & 44 to $147 \mathrm{IU} / \mathrm{L}$ & NS \\
\hline Bilirubin Direct & $2.44 \pm 12.48$ & $0.19 \pm 0.11$ & $0.1-0.4 \mathrm{mg} / \mathrm{dL}$ & NS \\
\hline Bilirubin Total & $0.56 \pm 0.37$ & $0.59 \pm 0.33$ & $0.2-1.2 \mathrm{mg} / \mathrm{dL}$ & NS \\
\hline Bilirubin Indirect & $0.41 \pm 0.26$ & $0.40 \pm 0.21$ & & NS \\
\hline GGT & $57.07 \pm 69.32$ & $39.00 \pm 40.15$ & $0-45 \mathrm{U} / \mathrm{L}$ & NS \\
\hline SGOT or AST & $41.70 \pm 64.92$ & $28.78 \pm 15.0$ & 5 to $40 \mathrm{U} / \mathrm{L}$ & NS \\
\hline SGPT or ALT & $60.73 \pm 121.77$ & $33.21 \pm 24.63$ & 7 to $56 \mathrm{U} / \mathrm{L}$ & NS \\
\hline
\end{tabular}

Data is expressed as mean \pm SD, DM+ HTN; diabetic hypertensive, DM; diabetes, GGT; Gamma-Glutamyl Transpeptidase, SGOT or AST; aspartate aminotransferase, SGPT or ALT; alanine aminotransferase, P value $<0.05$ is considered as significant.

Table 3: Showing comparison of liver function test parameters with extend of glycemic control

\begin{tabular}{|l|c|c|c|c|}
\hline Parameters & $\begin{array}{c}\text { Good control } \\
(\mathbf{n = 3 6})\end{array}$ & $\begin{array}{c}\text { Poor control } \\
(\mathbf{n = 6 1})\end{array}$ & $\begin{array}{c}\text { Uncontrolled } \\
(\mathbf{n = 4 4 )}\end{array}$ & $\begin{array}{c}\mathbf{P} \\
\text { value }\end{array}$ \\
\hline Alkaline Phosphate & $105.54 \pm 50.65$ & $98.41 \pm 32.98$ & $127.66 \pm 59.70$ & $\mathrm{~b}, \mathrm{c}$ \\
\hline Bilirubin Direct & $2.46 \pm 0.48$ & $0.16 \pm 0.09$ & $0.21 \pm 0.16$ & $\mathrm{a}, \mathrm{b}$ \\
\hline Bilirubin total & $0.64 \pm 0.28$ & $0.50 \pm 0.21$ & $0.64 \pm 0.21$ & $\mathrm{NS}$ \\
\hline Bilirubin Indirect & $0.43 \pm 0.16$ & $0.35 \pm 0.13$ & $0.44 \pm 0.9$ & $\mathrm{NS}$ \\
\hline GGT & $36.08 \pm 12.69$ & $42.46 \pm 14.67$ & $50.68 \pm 19.36$ & $\mathrm{~b}, \mathrm{c}$ \\
\hline SGOT & $29.29 \pm 12.36$ & $29.12 \pm 11.40$ & $61.25 \pm 39.05$ & $\mathrm{~b}, \mathrm{c}$ \\
\hline SGPT & $33.32 \pm 13.17$ & $34.94 \pm 18.32$ & $112.95 \pm 54.50$ & $\mathrm{~b}, \mathrm{c}$ \\
\hline
\end{tabular}

$\mathrm{P}<0.05$ considered as statistically significant as determined by ANOVA with post-hoc multiple comparison Bonferroni test; a- significant difference between good and poor control, b- significant difference between good and uncontrolled, c- significant difference between uncontrolled and poor control, GGT; GammaGlutamyl Transpeptidase, SGOT or AST; aspartate aminotransferase, SGPT or ALT; alanine aminotransferase 


\section{Discussion}

Reports have shown the higher incidence of abnormal liver function tests in patients with T2DM compared to patients without diabetes., 10 In present study we tried to compare the liver function test parameters in patients with diabetes (Group D) and hypertensive diabetes (Group HD). Reports have also shown that raised ALT is most common abnormality in diabetes patients. ${ }^{10}$

$\mathrm{Ni}$ et al performed a study on $81 \mathrm{~T} 2 \mathrm{DM}$ patients in Myanmar and reported that around 20\% of the patients were found to have elevated level of ALT and AST in T2DM patients. ${ }^{9}$ in present study also SGOT and SGPT were higher than the normal range in Group D which is consistence with the study done by Ni et al. In present study in Group $\mathrm{HD}$, none of the parameter was higher than the reference range, neglecting the role of hypertension in patients with diabetes on liver function test parameters.

Choudhary et al compared the liver function test parameters in 25 patients of T2DM and 25 patients of type 1 diabetes mellitus. Choudhary et al reported that SGOT, SGPT, ALP and GGT were significantly high in patients with T2DM as compared to T1DM. But no association was observed in bilirubin values between both the groups. ${ }^{11}$ In present study, GGT, SGOT and SGPT were higher than the normal along with the direct bilirubin, which was contrary to the study done by Choudhary et al. ${ }^{11}$ A study done by Salih on 55 blood sample of diabetic patients also reported raised AST and ALT parameters, which is in accordance with the present study data. ${ }^{1}$

Munazza et al studied liver function test on 50 pregnant women with preeclampsia after 20 weeks of gestation. Munazza et al reported increased level of serum bilirubin and liver enzymes such as ALT, AST and ALK in pregnant women with preeclampsia as compared to normal pregnant women. ${ }^{12}$ This showed that liver function test parameters are affected by increase in blood pressure in pregnant women, but such association of hypertension and liver function test parameters was not obtained in present study.
Gonem et al studied 959 patients to investigate liver function test, especially ALP, ALT and bilirubin level and observation are in accordance with the present study findings. ${ }^{13}$

Alkaline Phosphate, GGT, SGOT and SGPT were significantly higher in patients with uncontrolled diabetes as compared to patients with good control $(\mathrm{p}<0.05)$. Cho et al also reported that abnormal increase of liver enzyme such as GGT, SGOT and SGPT are the marker of hepatocellular injury. Increase level of these markers is also associated with increase insulin resistance, metabolic syndrome and worsening to type 2 diabetes mellitus. ${ }^{14}$

This raised level of liver function test might be the marker of non-alcoholic steatohepatitis (NASH) and insulin resistance. ${ }^{13}$ Presently, routine screening of liver function test is not being followed in patients with T2DM, but emerging evidence along with present study data suggests that abnormal liver function test might be the marker of insulin resistance and metabolic syndrome.

\section{Conclusion}

Abnormal liver function results were common among diabetes mellitus patients. In present study, liver function test parameters such as bilirubin direct, GGT, SGOT and SGPT were higher in patients with T2DM but comparable with patients with hypertensive diabetes mellitus. Abnormal liver function test are also a marker of uncontrolled diabetes mellitus. Tests for liver function could be done in patients with diabetes.

\section{References}

1. Salih DH. Study of Liver Function Tests and Renal Function Tests in diabetic type II patients. IOSR Journal of Applied Chemistry (IOSR-JAC) 2013; 3 (3):42-4.

2. Hams HE Elevated Liver Function Tests in Type 2 Diabetes. Clinical Diabetes 2005; 23(3): 215-9.

3. Chatila R, West AB. Hepatomegaly and abnormal liver tests due to glycogenosis in 
adults with diabetes. Medicine 1996; 75(6):327-33.

4. Levinthal G.N, Tavill A.S. Liver disease and diabetes mellitus. Clin Diabetes 1999; 17 (2): 1-20.

5. Lenfant C; National Education Program Working Group on High Blood Pressure in Pregnancy. Working Group Report on High blood pressure in pregnancy. J Clin Hypertens (Greenwich) 2001;3(2):75-88.

6. Wetzka B, Nusing R, Charnock DS, Janes Schafer W, Zahradni HP, Smith SK. Cycloxygenase-1-and 2 in a human placenta and placental bed after normal and preeclamptic pregnancies. Human Reprod 2008; 12:2313-20.

7. Mills JL, DerSimonian R, Raymond E, Morrow JD, Roberts LJ, Clemens JD, et al. Prostacyclin and thromboxane changes predating clinical onset of preeclampsia: a multicenter prospective study. JAMA 1999; 282 :356-62.

8. Wehbi M. Bilirubin. Medscape. http://emedicine.medscape.com/article/207 4068-overview. Accessed on 13 August 2016.

9. Ni H, Soe HHK, Htet A. Determinants of Abnormal Liver Function Tests in Diabetes Patients in Myanmar. International Journal of Diabetes Research 2012; 1(3): 36-41.

10. Harris E H. Elevated Liver Function Tests in Type 2 Diabetes. Clinical Diabetes 2005; 23 (3): 115-9.

11. Choudhary M, Jinger SK, Yogita, Gahlot G, Saxena R.Comparative study of liver function test in type-1 and type-2 diabetes mellitus. Indian J Sci Res 2014; 5 (2): 1437.

12. Munazza B, Raza N, Naureen A, Khan SA, Fatima F, Ayub $M$ et a. Liver Function Tests in Preeclampsia. J Ayub Med Coll Abbottabad 2011;23(4): 3-5.
13. Gonem S, Wall A, De P. Prevalence of abnormal liver function tests in patients with diabetes mellitus. Endocrine Abstracts 2007; 13: P157

14. Cho NH, Jang HC, Choi SH, Kim HR, Lee HK, Chan JCN et al. Abnormal Liver Function Test Predicts Type 2 Diabetes. Diabetes Care 2007; 30(10): 2566-8. 